Pacific Journal of Mathematics

EIGENVALUES OF SUMS OF HERMITIAN MATRICES 


\section{EIGENVALUES OF SUMS OF HERMITIAN MATRICES}

\section{ALFRED HORN}

Let $\alpha=\left(\alpha_{1}, \cdots, \alpha_{n}\right)$ and $\beta=\left(\beta_{1}, \cdots, \beta_{n}\right)$ be arbitrary nonincreasing sequences of real numbers. We consider the question: for which nonincreasing sequences $\gamma=\left(\gamma_{1}, \cdots, \gamma_{n}\right)$ do there exist Hermitian matrices $A$ and $B$ such that $A, B$ and $A+B$ have $\alpha, \beta$ and $\gamma$ respectively as their sequences of eigenvalues. Necessary conditions have been obtained by several authors including Weyl [4], Lidskii [3], Wielandt [5], and Amir-Moez [1]. Besides the obvious condition

$$
\gamma_{1}+\cdots+\gamma_{n}=\alpha_{1}+\cdots+\alpha_{n}+\beta_{1}+\cdots+\beta_{n},
$$

these conditions are linear inequalities of the form

$$
\gamma_{k_{1}}+\cdots+\gamma_{k_{r}} \leqq \alpha_{i_{1}}+\cdots+\alpha_{i_{r}}+\beta_{j_{1}}+\cdots \beta_{j_{r}},
$$

where $i, j$ and $k$ are increasing sequences of integers. As far as I know all other known necessary conditions are consequences of these inequalities. It is therefore natural to conjecture that the set $E$ of all possible $\gamma$ forms a convex subset of the hyperplane (1). The set $E$ has hitherto not been determined except in the simple cases $n=1,2$, and will not be determined in general here.

In $\S 2$, which is independent of $\S 1$, we are going to give a method of finding conditions of the form (2) which will yield many new ones. We shall find all possible inequalities (2) for $r=1,2$, and arbitrary $n$, and establish a large class of such inequalities for $r=3$. In $\S 1$, we use Lidskii's method to find a necessary condition on the boundary points of a subset $E^{\prime}$ of $E$. These results are used in $\S 3$ to determine the set $E$ for $n=3,4$. In addition a conjecture is given for $E$ in general.

If $x$ is a sequence, $x_{p}$ denotes the $p$ th component of $x$. If $A$ is a matrix, $A^{*}$ and $A^{\prime}$ denote the conjugate transpose and transpose of $A$. If $i$ is a sequence of integers such that $1 \leqq i_{1}<\cdots<i_{r} \leqq n$, by the complement of $i$ with respect to $n$ we mean the sequence obtained by deleting the terms of $i$ from the sequence $1,2, \cdots, n$. If $\alpha$ is a sequence of numbers, diag $\left(\alpha_{1}, \cdots, \alpha_{n}\right)$ denotes the diagonal matrix with diagonal $\alpha$. If $M$ and $N$ are matrices, $\operatorname{diag}(M, N)$ denotes the direct sum matrix

$$
\left(\begin{array}{cc}
M & 0 \\
0 & N
\end{array}\right)
$$

The inner product of the vectors $x$ and $y$ is written $(x, y) . \quad I_{r}$ is the

Received January 16, 1961. Part of this research was supported by a National Science Foundation grant. 
unit matrix of order $r$. Finally $\exp B$ denotes the $\operatorname{sum} \sum_{n=0}^{\infty} B^{n} / n !$.

1. Boundary points of $E^{\prime}$. In this section we are going to use methods introduced by Lidskii [3]. Lidskii gave sketchy proofs of his results and it is not obvious how to reconstruct his argument, see [5]. I will therefore derive the results of Lidskii which are needed. These are Theorem 1 and formula (18) below.

The set $E$ referred to in the introduction is the set of points $\gamma$ such that $\gamma_{1} \geqq \cdots \geqq \gamma_{n}$ and $\gamma$ is the sequence of eigenvalues of diag $\left(\alpha_{1}, \cdots\right.$, $\left.\alpha_{n}\right)+U^{*}$ diag $\left(\beta_{1}, \cdots, \beta_{n}\right) U$, where $U$ ranges over all unitary matrices. Fix $\alpha, \beta$, with $\alpha_{1}>\cdots>\alpha_{n}$ and $\beta_{1}>\cdots>\beta_{n}$, and let $E^{\prime}$ be the subset of $E$ obtained by letting $U$ range over real orthogonal matrices. To indicate the dependence of $E^{\prime}$ on $\alpha$ and $\beta$ we write $E^{\prime}\left(\alpha_{1}, \cdots, \alpha_{n} ; \beta_{1}\right.$, $\left.\cdots, \beta_{n}\right)$. Boundary points and interior points of $E^{\prime}$ are always taken with respect to the relative topology of the hyperplane (1).

THEOREM 1. If $\gamma$ is a boundary point of $E^{\prime}$ with distinct coordinates then there exist a positive integer $r<n$ and increasing sequences $i, j$, and $k$ of order $r$ such that

$$
\left(\gamma_{k_{1}}, \cdots, \gamma_{k_{r}}\right) \in E^{\prime}\left(\alpha_{i_{1}}, \cdots, \alpha_{i_{r}} ; \beta_{j_{1}}, \cdots, \beta_{j_{r}}\right)
$$

and

$$
\left(\gamma_{k^{\prime} 1}, \cdots, \gamma_{k^{\prime}{ }_{n-r}}\right) \in E^{\prime}\left(\alpha_{i^{\prime} i}, \cdots, \alpha_{i^{\prime} n-r} ; \beta_{j^{\prime} 1_{1}}, \cdots, \beta_{j^{\prime} n_{-r}}\right),
$$

where $i^{\prime}, j^{\prime}$ and $k^{\prime}$ are the complements of $i, j$ and $k$ with respect to $n$.

Proof. Let $U_{0}$ be a real orthogonal matrix such that diag $\left(\alpha_{1}, \cdots\right.$, $\left.\alpha_{n}\right)+U_{0}^{\prime}$ diag $\left(\beta_{1}, \cdots, \beta_{n}\right) U_{0}$ has eigenvalues $\gamma$. If $T=\left(t_{p q}\right)$ is a real anti-symmetric matrix, $\exp T$ is orthogonal. For sufficiently small values of $t_{p q}$, the eigenvalues $\lambda_{1}>\cdots>\lambda_{n}$ of

$$
\operatorname{diag}\left(\alpha_{1}, \cdots, \alpha_{n}\right)+U_{0}^{\prime} \exp (-T) B \exp (T) U_{0},
$$

where $B=\operatorname{diag}\left(\beta_{1}, \cdots, \beta_{n}\right)$, are distinct and determine a point of $E^{\prime}$. Let $A=U_{0} \operatorname{diag}\left(\alpha_{1}, \cdots, \alpha_{n}\right) U_{0}^{\prime}$, and let $x_{l}$ be a unit eigenvector of $A+$ $\exp (-T) B \exp T$ corresponding to the eigenvector $\lambda_{l}$ which varies continuously with $T$. We have

$$
A x_{\imath}+\exp (-T) B \exp (T) x_{l}=\lambda_{l} x_{l} .
$$

Using superscripts to denote derivatives with respect to $t_{p q}, p<q$, it follows that

$$
\begin{aligned}
A x_{l}^{p q}+\exp (-T) B \exp (T) x_{l}^{p q}+(\exp (-T) B \exp (T))^{p q} x_{l} & \\
& =\lambda_{l}^{p q} x_{l}+\lambda_{l} x_{l}^{p q}
\end{aligned}
$$


It is easily seen that $(\exp T)^{p q}$ reduces to $T^{p q}$ when $T=0$. Hence when $T=0,(\exp (-T) B \exp T)^{p q},=\left(\beta_{p}-\beta_{q}\right) Z^{p q}$, where $Z^{p q}$ is the matrix whose $(p, q)$ and $(q, p)$ entries are 1 and whose other entries are 0 . Since $x_{l}$ is a unit vector, $\left(x_{l}, x_{l}^{x q}\right)=0$. Therefore by (3),

$$
\left(A x_{l}, x_{l}^{x q}\right)+\left(\exp (-T) B \exp (T) x_{l}, x_{l}^{x q}\right)=0 .
$$

Taking the inner product of (4) with $x_{\imath}$ we find by (5) and the symmetry of $A$ and $B$,

$$
\lambda_{l}^{p q}=\left((\exp (-T) B \exp T)^{p q} x_{l}, x_{l}\right) .
$$

iSetting $T=0$,

$$
\gamma_{l}^{p q}=2\left(\beta_{p}-\beta_{q}\right) w_{l p} w_{l q},
$$

where $w_{l}$ and $\gamma_{l}^{q q}$ denote the values of $x_{\imath}$ and $\lambda_{l}^{\gamma q}$ when $T=0$. If $\gamma$ is not an interior point of $E^{\prime}$ the rank of the $n$ by $n(n-1) / 2$ matrix $G=\left(\gamma_{l}^{q q}\right)$ must be less than $n-1$. Now let $D=\left(w_{l p} w_{l q}\right)$ be the $n$ by $n(n-1)$ matrix whose rows are indexed by $l$, where $1 \leqq l \leqq n$, and whose columns are indexed by $(p, q)$, where $p$ and $q$ vary over the range $1 \leqq p \leqq n, 1 \leqq q \leqq n$, and $p \neq q$ rather than $p<q$. Clearly $D$, and hence $D D^{\prime}$, has the same rank as $G$. If $F$ is the square matrix $\left(w_{l_{m}}^{2}\right)$ of order $n$, then $D D^{\prime}=I-F F^{\prime}$. Thus if $\operatorname{rank} D<n-1, F F^{\prime}$ has 1 as a multiple eigenvalue. Since $F F^{\prime}$ is stochastic, it follows that $F F^{\prime}$ is decomposable [2, pp. 47 and 73]. That is to say, $F F^{\prime}=P \operatorname{diag}(M, N) P^{\prime}$, where $M$ and $N$ are square matrices and $P$ is a permutation matrix. Let

$$
F=P\left(\begin{array}{cc}
G & H \\
J & K
\end{array}\right) P^{\prime}
$$

be the decomposition of $F$ corresponding to that of $F F^{\prime}$. Then $G J^{\prime}+$ $H K^{\prime}=0$. Since the entries of $F$ are nonnegative, we have $G J^{\prime}=H K^{\prime}=$ 0 . It follows that if a column of $G$ contains a nonzero term then all terms of the corresponding column of $J$ vanish, and similarly for $H$ and $K$. Moving all nonzero columns of $G$ and $H$ to the left, we find

$$
F=P\left(\begin{array}{cc}
S_{1} & 0 \\
0 & S_{2}
\end{array}\right) R
$$

where $R$ is another permutation matrix. Since $F$ is doubly stochastic, $S_{1}$ and $S_{2}$ must be square matrices. If $W=\left(w_{l m}\right)$, then $W=P\left(\begin{array}{cc}W_{1} & 0 \\ 0 & W_{2}\end{array}\right) R$, where $W_{1}$ and $W_{2}$ are square. Setting $\Gamma=\operatorname{diag}\left(\gamma_{1}, \cdots, \gamma_{n}\right)$, we have $A+B=W^{\prime} \Gamma W$. Therefore $R A R^{\prime}+R B R^{\prime}=C$, where $C=$

\footnotetext{
1 The following argument is due to Robert Steinberg.
} 
$\operatorname{diag}\left(W_{1}^{\prime}, W_{2}^{\prime}\right) P^{\prime} \Gamma P \operatorname{diag}\left(W_{1}, W_{2}\right)$. Let $j$ and $k$ be such that $R B R^{\prime}=\operatorname{diag}\left(\beta_{j_{1}}\right.$, $\left.\cdots, \beta_{\jmath_{n}}\right)$ and $P^{\prime} \Gamma P=\operatorname{diag}\left(\gamma_{k_{1}}, \cdots, \gamma_{k_{n}}\right)$. If $W_{1}$ is of order $r$, then $C=$ $\operatorname{diag}\left(C_{1}, C_{2}\right)$, where $C_{1}$ has eigenvalues $\gamma_{k_{1}}, \cdots, \gamma_{k_{r}}$ and $C_{2}$ has eigenvalues. $\gamma_{k_{r+1}}, \cdots, \gamma_{k_{n}}$. Therefore $R A R^{\prime}=\operatorname{diag}\left(A_{1}, A_{2}\right)$, where $A_{1}+\operatorname{diag}\left(\beta_{1_{1}}, \cdots\right.$, $\left.\beta_{j_{r}}\right)=C_{1}$ and $A_{2}+\operatorname{diag}\left(\beta_{j_{r+1}}, \cdots, \beta_{j_{n}}\right)=C_{2}$. This completes the proof.

If $M=\left(m_{i j}\right), 1 \leqq i \leqq r, 1 \leqq j \leqq r$ is a matrix of order $r$ and $N=$ $\left(n_{k l}\right), r+1 \leqq k \leqq n, r+1 \leqq l \leqq n$ is a matrix of order $\mathrm{n}-r$, we define $M \times N$ to be the matrix $\left(m_{i j} n_{k l}\right)$ of order $r(n-r)$ whose rows are indexed by pairs $(i, k)$ and whose columns are indexed by pairs $(j, l)$. This product is left and right distributive and $(M \times N)^{\prime}=M^{\prime} \times N^{\prime}$. Also $\left(M_{1} \times N_{1}\right)\left(M_{2} \times N_{2}\right)=\left(M_{1} M_{2} \times N_{1} N_{2}\right)$. We set $M \ominus N=\left(M \times I_{n-r}\right)-$ $\left(I_{r} \times N\right)$. It follows from these remarks that if $W_{1}$ and $W_{2}$ are orthogonal then so is $W_{1} \times W_{2}$ and

$$
\left(W_{1}^{\prime} M W_{1}\right) \ominus\left(W_{2}^{\prime} N W_{2}\right)=\left(W_{1} \times W_{2}\right)^{\prime}(M \ominus N)\left(W_{1} \times W_{2}\right) .
$$

The index of a real symmetric matrix is the number of its positive eigenvalues.

Lemma 1. If $M, N$, and $M+N$ are nonsingular real symmetric matrices then index $M+$ index $N=$ index $(M+N)+$ index $\left(M^{-1}+N^{-1}\right)$.

Proof. ${ }^{2}$ We have $M^{-1}+N^{-1}=N^{-1}(N+M) M^{-1}$, so that $M^{-1}+N^{-1}$ is nonsingular. Also

$$
\left(\begin{array}{cc}
I & I \\
M^{-1} & -N^{-1}
\end{array}\right)\left(\begin{array}{cc}
M & 0 \\
0 & N
\end{array}\right)\left(\begin{array}{cc}
I & M^{-1} \\
I & -N^{-1}
\end{array}\right)\left(\begin{array}{cc}
M+N & 0 \\
0 & M^{-1}+N^{-1}
\end{array}\right) .
$$

The result now follows by the Law of Inertia.

THEOREM 2. Let $\gamma$ be a boundary point of $E^{\prime}$ with distinct coordinates. Then there exist sequences $i, j$ and $k$ satisfying the conclusion. of Theorem 1 and such that

$$
i_{1}+\cdots+i_{r}+j_{1}+\cdots+j_{r}=k_{1}+\cdots+k_{r}+r(r+1) / 2 .
$$

Proof. Using a slight change of notation, we have seen that there exist permutations $i, j$ and $k$ of $(1, \cdots, n)$ and real symmetric matrices $A_{1}, A_{2}, B_{1}, B_{2}, C_{1}, C_{2}$ such that $A_{1}$ has eigenvalues $\alpha_{i_{1}}, \cdots, \alpha_{i_{r}}, A_{2}$ has eigenvalues $\alpha_{i_{r+1}}, \cdots, \alpha_{i_{n}}, B_{1}=\operatorname{diag}\left(\beta_{j_{1}}, \cdots, \beta_{j_{r}}\right), B_{2}=\operatorname{diag}\left(\beta_{j_{r+1}}, \cdots, \beta_{j_{n}}\right)$, $C_{1}$ has eigenvalues $\gamma_{k_{1}}, \cdots, \gamma_{k_{r}}, C_{2}$ has eigenvalues $\gamma_{k_{r+1}}, \cdots, \gamma_{k_{n}}$, and $A+B=C$, where $A=\operatorname{diag}\left(A_{1}, A_{2}\right), B=\operatorname{diag}\left(B_{1}, B_{2}\right) C=\operatorname{diag}\left(C_{1}, C_{2}\right)$. We also assume $i_{1}<\cdots<i_{r}$ and $i_{r+1}<\cdots<i_{n}$, and similarly for the $j$ 's and $k$ 's. We set $\bar{\alpha}_{l}=\alpha_{i_{l}}, \bar{\beta}_{l}=\beta_{j_{l}}$ and $\bar{\gamma}_{l}=\gamma_{k_{l}}, 1 \leqq l \leqq n$. Let.

2 This simple proof is due to Robert Steinberg. 
$T=\left(t_{p q}\right)$ be a real anti-symmetric matrix and let $\lambda_{1}>\cdots>\lambda_{n}$ be the eigenvalues of $A+\exp (-T) B \exp T$. If $x_{1}, \cdots, x_{n}$ is a real orthonormal system of corresponding eigenvectors, we let $w_{l}$ and $w_{l}^{p q}$ be the values of $x_{k_{l}}$ and $x_{k_{l}}^{p q}$ when $T=0$, where $x_{l}^{p q}$ denotes the derivative of $x_{l}$ with respect to $t_{p q}, p<q$. If $W$ is the matrix whose rows are $w_{1}, \cdots, w_{n}$, then $W=\operatorname{diag}\left(W_{1}, W_{2}\right)$ and $C_{1}=W_{1}^{\prime} \Gamma_{1} W_{1}, C_{2}=W_{2}^{\prime} \Gamma_{2} W_{2}$, where $\quad \Gamma_{1}=\operatorname{diag}\left(\gamma_{k_{1}}, \cdots, \gamma_{k_{r}}\right), \quad \Gamma_{2}=\operatorname{diag}\left(\gamma_{k_{r+1}}, \cdots, \gamma_{k_{n}}\right)$, Clearly $\lambda_{k_{l}}$ reduces to $\bar{\gamma}_{l}$ when $T=0$, and we let $\bar{\gamma}_{l}^{p q}$ be the value of $\lambda_{k_{l}}^{p q}=\partial \lambda_{k_{l}} / \partial t_{p q}$ when $T=0$.

Starting from the equation

$$
A x_{k_{l}}+(\exp (-t) B \exp T) x_{k l}=\lambda_{k l} x_{k_{l}}
$$

we find

$$
\begin{aligned}
A x_{k_{l}}^{p q}+(\exp (-T) B \exp T) x_{k l}^{p q}+(\exp (-T) B \exp T)^{p q} x_{k_{l}} \\
=\lambda_{k_{l}}^{p q} x_{k_{l}}+\lambda_{k_{l}} x_{k l}^{p q}
\end{aligned}
$$

As in Theorem 1 it follows that

$$
\lambda_{k_{l}}^{p q}=\left((\exp (-T) B \exp T)^{p q} x_{k_{l}}, x_{k_{l}}\right)
$$

and therefore

$$
\bar{\gamma}_{l}^{p q}=2\left(\bar{\beta}_{p}-\bar{\beta}_{q}\right) w_{l p} w_{l q} .
$$

We are going to test $\sigma=\lambda_{k_{1}}+\cdots+\lambda_{k_{r}}$ for a local extreme at $T=0$. If $p$ and $q$ are $\leqq r$, then $\exp T$ has the form diag $\left(\exp T_{1}, 0\right)$ when $t_{u v}=0$ for $(u, v) \neq(p, q)$, and hence $\sigma$ remains constant for $t_{p q}$ in a neighborhood of 0 . Therefore all partial derivatives of $\sigma$ with respect to $t_{p q}$ vanish at the origin when $p<q \leqq r$, and similarly when $r<p<q$. By (11), $\sigma^{p q}=0$ at $T=0$ when $p \leqq r<q$, since the last $n-r$ components of $w_{l}$ are 0 when $1 \leqq l \leqq r$. We now calculate $\lambda_{k l}^{p q u v}$ at $T=0$ when

$$
1 \leqq p \leqq r<q \leqq n, \quad 1 \leqq u \leqq r<v \leqq n, \quad 1 \leqq l \leqq r .
$$

Differentiation of (10) yields

$$
\begin{aligned}
\lambda_{k_{l}}^{p q, u v}=\left((\exp (-T) B \exp T)^{p q, u v} x_{k_{l}}, x_{k_{l}}\right) & \\
& +2\left((\exp (-T) B \exp T)^{p q} x_{k_{l}}^{u v}, x_{k_{l}}\right) .
\end{aligned}
$$

It is easily seen that when $T=0$

$$
\begin{aligned}
(\exp (-T) B \exp T)^{p q, u v} & =-\left(T^{p q} B T^{u v}+T^{u v} B T^{p q}\right) \\
& +\frac{1}{2} B\left(T^{p q} T^{u v}+T^{u v} T^{p q}\right)+\frac{1}{2}\left(T^{p q} T^{u v}+T^{u v} T^{p q}\right) B
\end{aligned}
$$

Considering only the cases (12), a straightforward calculation shows that 
when $T=0$,

$$
\begin{array}{rlrl}
\left((\exp (-T) B \exp T)^{p q, u v} x_{k_{l}}, x_{k_{l}}\right)=0 & \text { for } & p \neq u, q \neq v \\
& =\left(2 \bar{\beta}_{q}-\bar{\beta}_{p}-\bar{\beta}_{u}\right) w_{l p} w_{l u} & \text { for } & p \neq u, q=v \\
& =\left(2 \bar{\beta}_{p}-\bar{\beta}_{q}-\bar{\beta}_{v}\right) w_{l q} w_{l v} & \text { for } & p=u, q \neq v \\
& =-2\left(\bar{\beta}_{p}-\bar{\beta}_{q}\right)\left(w_{l p}^{2}-w_{l p}^{2}\right) & \text { for } & p=u, q=v .
\end{array}
$$

Recalling that $w_{l q}=0$ for $l \leqq r<q$, we find that when $T=0$,

$$
\begin{aligned}
\sum_{l=1}^{r}\left((\exp (-T) B \exp T)^{p q, u v} x_{k_{l}}, x_{k_{l}}\right) & =-2\left(\bar{\beta}_{p}-\bar{\beta}_{q}\right) \text { for } p=u, q=v \\
& =0 \text { otherwise. }
\end{aligned}
$$

The second term on the right of (13) reduces when $T=0$ to

$$
2\left(\bar{\beta}_{p}-\bar{\beta}_{q}\right) w_{l q}^{u v} w_{l p} .
$$

To compute $w_{l q}^{u v}$, rewrite (9) in the form

$$
\begin{aligned}
(A+\exp (-T) B \exp & \left.T-\lambda_{k_{l}} I_{n}\right) x_{k_{l}}^{u v} \\
= & -(\exp (-T) B \exp T)^{u v} x_{k_{l}}+\lambda_{k_{l}}^{u v} x_{k_{l}} .
\end{aligned}
$$

Setting $T=0$ and using (11), we find, since $w_{l v}=0$,

$$
\left(C-\bar{\gamma}_{l} I_{n}\right) w_{l}^{u v}=-\left(\bar{\beta}_{u}-\bar{\beta}_{v}\right) y,
$$

where $y$ is the vector such that $y_{u}=w_{l v}=0, y_{v}=w_{l u}$ and $y_{m}=0$ for $m \neq u, m \neq v$. Therefore

$$
w_{l q}^{u v}=\left(\bar{\beta}_{u}-\bar{\beta}_{v}\right)\left(\left(\bar{\gamma}_{l} I_{n}-C\right)^{-1} y\right)_{q} .
$$

Since $q>r$, and $C=\operatorname{diag}\left(C_{1}, C_{2}\right)$, we may replace $C$ by $C_{2}$ and $I_{n}$ by $I_{n-r}$. Thus

$$
w_{l q}^{u v}=\left(\bar{\beta}_{u}-\bar{\beta}_{v}\right) d_{q v} w_{l u},
$$

where $d_{q v}$ is the $(q, v)$ entry of $\left(\bar{\gamma}_{l} I_{n-r}-C_{2}\right)^{-1}$. Now

$$
\left(\bar{\gamma}_{l} I_{n-r}-C_{2}\right)^{-1}=\left(W_{2}^{\prime}\left(\bar{\gamma}_{l} I_{n-r}-\Gamma_{2}\right) W_{2}\right)^{-1} .
$$

Therefore

$$
d_{q v}=\sum_{m=r+1}^{n} \frac{w_{m q} w_{m v}}{\bar{\gamma}_{l}-\bar{\gamma}_{m}}
$$

Combining (13), (14), (15), (16), and (17), we find at $T=0$

$$
\begin{gathered}
\sigma^{p q, u v}=2\left(\bar{\beta}_{p}-\bar{\beta}_{q}\right)\left(\bar{\beta}_{u}-\bar{\beta}_{v}\right) \sum_{i=1}^{r} \sum_{m=r+1}^{n} \frac{w_{l p} w_{l u} w_{m q} w_{m v}}{\bar{\gamma}_{l}-\bar{\gamma}_{m}} \\
-2 \delta_{u v}^{p q}\left(\bar{\beta}_{p}-\bar{\beta}_{q}\right)
\end{gathered}
$$


where $\delta_{u v}^{p q}=1$ when $(p, q)=(u, v)$, and $=0$ otherwise.

We must now determine the index of the matrix $G=\left(\sigma^{p q, u v}\right)_{T=0}$ of order $r(n-r)$ whose rows and columns are indexed by pairs $(p, q)$ and $(u, v)$ satisfying (12).

The double sum on the right of $(18)$ is the $(p q, u v)$ entry of

$$
\left(W_{1} \times W_{2}\right)^{\prime}\left(\Gamma_{1} \ominus \Gamma_{2}\right)^{-1}\left(W_{1} \times W_{2}\right)=\left(\left(W_{1} \times W_{2}\right)^{\prime}\left(\Gamma_{1} \ominus \Gamma_{2}\right)\left(W_{1} \times W_{2}\right)\right)^{-1} .
$$

By (7) this reduces to

$$
\left(C_{1} \ominus C_{2}\right)^{-1}=\left(\left(A_{1}+B_{1}\right) \ominus\left(A_{2}+B_{2}\right)\right)^{-1}=\left(\left(A_{1} \ominus A_{2}\right)+\left(B_{1} \ominus B_{2}\right)\right)^{-1} .
$$

Therefore by (18)

$$
\begin{aligned}
\frac{1}{2} G & =\left(B_{1} \ominus B_{2}\right)\left(\left(A_{1} \ominus A_{2}\right)+\left(B_{1} \ominus B_{2}\right)\right)^{-1}\left(B_{1}-B_{2}\right) \ominus\left(B_{1} \ominus B_{2}\right) \\
& =\left(B_{1} \ominus B_{2}\left(\left(\left(A_{1} \ominus A_{2}\right)+\left(B_{1} \ominus B_{2}\right)\right)^{-1}-\left(B_{1} \ominus B_{2}\right)^{-1}\right)\left(B_{1} \ominus B_{2}\right) .\right.
\end{aligned}
$$

Thus $G$ has the same index as $\left(\left(A_{1} \ominus A_{2}\right)+\left(B_{1} \ominus B_{2}\right)\right)^{-1}-\left(B_{1} \ominus B_{2}\right)^{-1}$. Applying Lemma 1 with $M=\left(\left(A_{1} \ominus A_{2}\right)+\left(B_{1} \ominus B_{2}\right)\right), N=-\left(B_{1} \ominus B_{2}\right)$,

$$
\text { index } \begin{aligned}
G= & \operatorname{index}\left(\left(C_{1} \ominus C_{2}\right)^{-1}-\left(B_{1} \ominus B_{2}\right)^{-1}\right) \\
= & \operatorname{index}\left(C_{1} \ominus C_{2}\right)+\operatorname{index}-\left(B_{1} \ominus B_{2}\right)-\operatorname{index}\left(A_{1} \ominus A_{2}\right) \\
& =r(n-r)+\operatorname{index}\left(C_{1} \ominus C_{2}\right)-\operatorname{index}\left(B_{1} \ominus B_{2}\right) \\
& \quad-\operatorname{index}\left(A_{1} \ominus A_{2}\right) .
\end{aligned}
$$

Thus $G$ is positive definite if and only index $\left(C_{1} \ominus C_{2}\right)=\operatorname{index}\left(A_{1} \ominus A_{2}\right)+$ index $\left(B_{1} \ominus B_{2}\right)$, and $G$ is negative definite if and only if neg $\left(C_{1} \ominus C_{2}\right)=$ neg $\left(A_{1} \ominus A_{2}\right)+$ neg $\left(B_{1} \ominus B_{2}\right)$, where neg $H$ is the number of negative eigenvalues of $H$. Next we determine

$$
\operatorname{neg}\left(B_{1} \ominus B_{2}\right)=\operatorname{neg} \operatorname{diag}\left(\beta_{j_{1}}-\beta_{j_{r+1}}, \cdots, \beta_{j_{1}}-\beta_{j_{n}}, \cdots, \beta_{j_{r}}-\beta_{j_{n}}\right) .
$$

Among the numbers $j_{r+1}, \cdots, j_{n}$, there are $j_{1}-1$ terms $<j_{1}, j_{2}-2$ terms $<$ $j_{2}$, etc. Hence neg $\left(B_{1} \ominus B_{2}\right)=j_{1}+\cdots+j_{r}-r(r+1) / 2$. Similarly neg $\left(A_{1} \ominus A_{2}\right)=i_{1}+\cdots+i_{r}-r(r+1) / 2$, and neg $\left(C_{1} \ominus C_{2}\right)=k_{1}+\cdots+$ $k_{r}-r(r+1) / 2$. Thus $G$ is negative definite if and only if

$$
i_{1}+\cdots+i_{r}+j_{1}+\cdots+j_{r}=k_{1}+\cdots+k_{r}+r(r+1) / 2,
$$

and $G$ is positive definite if and only if

$$
\begin{aligned}
i_{r+3}+\cdots+i_{n}+i_{r+1}+\cdots+j_{n}=k_{r+1} & +\cdots+k_{n} \\
& +(n-r)(n-r+1) / 2 .
\end{aligned}
$$

By Theorem 1 the boundary points of $E^{\prime}$ lie on a finite number of hyperplanes of the form

$$
\gamma_{k_{1}}+\cdots+\gamma_{k_{r}}=\alpha_{i_{1}}+\cdots+\alpha_{i_{r}}+\beta_{j_{1}}+\cdots+\beta_{j_{r}} .
$$


The hyperplane

$$
\gamma_{k_{1}^{\prime}}+\cdots+\gamma_{k_{n-r}^{\prime}}=\alpha_{i_{1}^{\prime}},+\cdots+\alpha_{i_{n-r}^{\prime}}+\beta_{j_{1}^{\prime}}+\cdots \beta_{j_{n-r}^{\prime}}
$$

intersects the hyperplane (1) in the same set. If $\gamma$ lies on only one of these hyperplanes (21) and does not satisfy (19) or (20), then in every small sphere about $\gamma$ there exist points of $E^{\prime}$ on both sides of the hyperplane (21). Therefore $E^{\prime}$ must fill the sphere, for otherwise there would be boundary points of $E^{\prime}$ inside the sphere and off the hyperplane (21). This being impossible, $\lambda$ must satisfy (19) or (20). Now suppose $\gamma$ lies on several hyperplanes (21), and (19) and (20) both fail for each of these hyperplanes. By continuity the quadratic form $G$ is not definite for all points near $\gamma$ which satisfy the conclusion of Theorem 1. Therefore in a neighborhood of $\gamma$ all points of $E^{\prime}$ lying on only one hyperplane (21) are interior points of $E^{\prime}$. Therefore $\gamma$ cannot be a boundary point of $E^{\prime}$, since $E^{\prime}$ is the closure of its interior, and a finite union of linear varieties of deficiency $\geqq 2$ cannot separate the interior of a sphere. The proof is complete.

2. Inequalities. This section is independent of $\S 1$. If $i, j$ and $k$ are increasing sequences of integers of order $r$ and (2) holds for the eigenvalues of $A+B$ for any Hermitian $A, B$ with arbitrary eigenvalues $\alpha_{1} \geqq \cdots \geqq \alpha_{n}$ and $\beta_{1} \geqq \cdots \geqq \beta_{n}$, we write $(i ; j ; k) \in S_{r}^{n}$. If

$$
\gamma_{k_{1}}+\cdots+\gamma_{k_{r}} \geqq \alpha_{i_{1}}+\cdots+\alpha_{i_{n}}+\beta_{j_{1}}+\cdots+\beta_{j_{r}}
$$

for any such $A, B$ we write $(i ; j ; k) \in \widetilde{S}_{r}^{n}$.

\section{THEOREM 3. The following conditions are equivalent:}

(i ) $(i ; j ; k) \in S_{r}^{n}$

(ii) $\left(n-i_{r}+1, \cdots, n-i_{1}+1 ; n-j_{r}+1, \cdots, n-j_{1}+1 ; n-k_{r}+1\right.$, $\left.\cdots, n-k_{1}+1\right) \in \widetilde{S}_{r}^{n}$

(iii) $\left(k_{1}, \cdots, k_{r} ; n-j_{r}+1, \cdots, n-j_{1}+1 ; i_{1}, \cdots, i_{r}\right) \in \widetilde{S}_{r}^{n}$

(iv) $\left(i^{\prime} ; j^{\prime} ; k^{\prime}\right) \in \widetilde{S}_{n-r}^{n}$, where $i^{\prime}, j^{\prime}, k^{\prime}$ are the complements of $i, j, k$ with respect to $n$.

Proof. The equation $A+B=C$ may be written $-A-B=-C$ or $A=C-B$. This proves the equivalence of (i) with (ii) and (iii). The equivalence of (i) and (iv) is immediate by the trace Condition (1).

If $A$ is a Hermitian matrix with eigenvalues $\alpha_{1} \geqq \cdots \geqq \alpha_{n}$ and $M$ is a linear subspace of dimension $n-1$, let $A_{\mu}$ be the transformation $P A$ with domain restricted to $M$, where $P$ is the orthogonal projection on $M . \quad A_{M}$ is a Hermitian transformation on $M$ to $M$ and $(A+B)_{M}=$ $A_{M}+B_{M}$. It is well known that the eigenvalues $\alpha_{p}^{\prime}$ of $A_{M}$ separate those of $A$, that is $\alpha_{p+1} \leqq \alpha_{p}^{\prime} \leqq \alpha_{p}$ for $1 \leqq p \leqq n-1$. If $\left(x_{p}\right)$ is an 
orthonormal sequence of eigenvectors corresponding to $\left(\alpha_{p}\right)$ and if $M$ contains $x_{1}, \cdots, x_{m}$, then $\alpha_{p}^{\prime}=\alpha_{p}$ for $1 \leqq p \leqq m$. This is an immediate consequence of the minimax principle, since $\left(A_{M} x, x\right)=(A x, x)$ for $x \in M$. Dually if $M$ contains $x_{m+1}, \cdots, x_{n}$, then $\alpha_{p}^{\prime}=\alpha_{p+1}$ for $m \leqq p \leqq n-1$. The next theorem shows that $S$ is essentially independent of $n$.

Theorem 4. If $(i ; j ; k) \in S_{r}^{n}$ for some $n$, then $i_{p} \leqq k_{p}$ and $j_{p} \leqq k_{p}$ for all $p$, and $(i ; j ; k) \in S_{r}^{n}$ for all $n \geqq k_{r}$.

Proof. Suppose $(i ; j ; k) \in S_{r}^{n}$ for some $n$. Considering the case $\beta=0$, it is clear that $i_{p} \leqq k_{p}$ and $j_{p} \leqq k_{p}$ for all $p$. If $A$ and $B$ are of order $k_{r}$, the identity $\operatorname{diag}(A,-\lambda I)+\operatorname{diag}(B,-\lambda I)=\operatorname{diag}(A+B,-2 \lambda I)$ for large $\lambda$ shows that $(i ; j ; k) \in S_{r}^{k}$. It remains to prove $(i ; j ; k) \in S_{r}^{n+1}$. Let $A$ and $B$ be of order $n+1$ with eigenvalues $\left(\alpha_{p}\right),\left(\beta_{p}\right)$, and let $\left(z_{p}\right)$ be an orthonormal sequence of eigenvectors of $A+B$ corresponding to the eigenvalues $\left(\gamma_{p}\right)$. Let $M$ be the subspace spanned by $z_{1}, \cdots, z_{n}$. Letting $\left(\alpha_{p}^{\prime}\right),\left(\beta_{p}^{\prime}\right)$ and $\left(\gamma_{p}^{\prime}\right)$ be the eigenvalues of $A_{M}, B_{M}$ and $(A+B)_{M}$, we have by hypothesis

$$
\gamma_{j_{1}}^{\prime}+\cdots+\gamma_{k_{r}}^{\prime} \leqq \alpha_{i_{1}}^{\prime}+\cdots+\alpha_{i_{r}}^{\prime}+\beta_{j_{1}}^{\prime}+\cdots+\beta_{j_{r}}^{\prime} .
$$

But $\gamma_{k_{p}}^{\prime}=\gamma_{k_{p}}, \alpha_{i_{p}}^{\prime} \leqq \alpha_{i_{p}}$ and $\beta_{j_{p}}^{\prime} \leqq \beta_{j_{p}}$ for $1 \leqq p \leqq r$. Therefore $(1 ; j ; k) \in S_{r}^{n+1}$.

THEOREM 5. If $(i ; j ; k) \in S_{r}^{n}$ and $u, v$ and $w$ are integers such that $r+1 \geqq u \geqq 1, r+1 \geqq v \geqq 1$ and $r \geqq w \geqq 1$, and if $i_{u}+j_{v} \geqq k_{w-1}+$ $k_{r}+2$ then $\left(i_{1}, \cdots, i_{u-1}, i_{u}+1, \cdots, i_{r}+1 ; j_{1} \cdots, j_{v-1}, j_{v}+1, \cdots, j_{r}+\right.$ $\left.1 ; k_{1}, \cdots, k_{w-1}, k_{w}+1, \cdots, k_{r}+1\right) \in S_{r}^{n+1}$. Here $k_{0}=0$ and $i_{r+1}=j_{r+1}=$ $k_{r}+1$ by definition. ${ }^{3}$ In particular, $\left(i_{1}+1, \cdots, i_{r}+1 ; j_{1}, \cdots, j_{r} ; k_{1}+\right.$ $\left.1, \cdots, k_{r}+1\right) \in S_{r}^{n+1}$.

Proof. By Theorem 4 we may assume $n=k_{r}$. Let $\left(x_{p}\right),\left(y_{p}\right)$ and $\left(z_{p}\right), 1 \leqq p \leqq n+1$, be orthonormal sequences of eigenvectors corresponding to the eigenvalues $\left(\alpha_{p}\right),\left(\beta_{p}\right)$ and $\left(\gamma_{p}\right)$ of $A, B$ and $A+B$. Since $i_{u}+j_{v} \geqq k_{w-1}+n+2$, there exists an $n$ dimensional subspace $M$ containing the vectors $x_{p}, i_{u}+1 \leqq p \leqq n+1$, the vectors $y_{p}, j_{v}+1 \leqq p \leqq n+1$, and the vectors $z_{p}, 1 \leqq p \leqq k_{w-1}$. Let $\left(\alpha_{p}^{\prime}\right),\left(\beta_{p}^{\prime}\right)$, and $\left(\gamma_{p}^{\prime}\right)$ be the eigenvalues of $A_{M}, B_{M}$, and $(A+B)_{M}$. By hypothesis

$$
\gamma_{k_{1}}^{\prime}+\cdots+\gamma_{k_{r}}^{\prime} \leqq \alpha_{i_{1}}^{\prime}+\cdots+\alpha_{i_{r}}^{\prime}+\beta_{j_{1}}^{\prime}+\cdots+\beta_{j_{r}}^{\prime} .
$$

The theorem now follows because $\gamma_{p}^{\prime}=\gamma_{p}$ for $1 \leqq p \leqq k_{w-1}, \gamma_{p+1} \leqq \gamma_{p}^{\prime}$ for $k_{w} \leqq p \leqq n, \alpha_{p}^{\prime} \leqq \alpha_{p}$ for $1 \leqq p \leqq i_{u-1}, \alpha_{p}^{\prime}=\alpha_{p+1}$ for $i_{u} \leqq p \leqq n, \beta_{p}^{\prime} \leqq \beta_{p}$ for $1 \leqq p \leqq j_{v-1}$ and $\beta_{p}^{\prime}=\beta_{p+1}$ for $j_{v} \leqq p \leqq n$.

${ }^{3}$ This theorem was suggested by a special case which was pointed out to me by Alan J. Hoffman. 
Theorem 5 yields a simple proof of the following theorem due to Lidskii.

Theorem 6 [3]. If $1 \leqq p_{1}<\cdots<p_{r} \leqq n$, then $\left(p_{1}, \cdots, p_{r} ; 1, \cdots, r\right.$; $\left.p_{1}, \cdots, p_{r}\right) \in S_{r}^{n}$.

Proof. Obviously $(1, \cdots, r ; 1, \cdots, r ; 1, \cdots, r) \in S_{r}^{n}$. Using Theorem $5 p_{1}-1$ times with $u=w=1, v=r+1$, we find $\left(p_{1}, p_{1}+1, \cdots, p_{1}+\right.$ $\left.r-1, ; 1, \cdots, r: p_{1}, p_{1}+1, \cdots, p_{1}+r-1\right) \in S_{r}^{p_{1}+r-1}$. Such use of Theorem 5 is justified since $i_{1}+j_{r+1}=i_{1}+k_{r}+1 \geqq k_{r}+2=k_{0}+k_{r}+2$ at each stage. We may now apply Theorem $5 p_{2}-\left(p_{1}+1\right)$ times with $u=w=2$, $v=r+1$ since at each stage $i_{2}+j_{r+1}=i_{2}+k_{r}+1 \geqq i_{1}+k_{r}+2=$ $k_{1}+k_{r}+2$. The result is

$$
\begin{aligned}
\left(p_{1}, p_{2}, p_{2}+1, \cdots, p_{2}+r\right. & -2 ; 1, \cdots, r ; p_{1}, p_{2}, p_{2} \\
& \left.+1, \cdots, p_{2}+r-2\right) \in S_{r}^{p_{2}+r-2} .
\end{aligned}
$$

Continuing in this way we find

$$
\left(p_{1}, \cdots, p_{r} ; 1, \cdots, r ; p_{1}, \cdots, p_{r}\right) \in S_{r}^{p_{r}} .
$$

By Theorem 4 the proof is complete.

THEOREM 7. $\left(i_{1} ; j_{1} ; k_{1}\right) \in S_{1}^{n}$ for $n \geqq k_{1}$ if and only if $1 \leqq i_{1} \leqq k_{1}$, $1 \leqq j_{1} \leqq k_{1}$, and $i_{1}+j_{1}=k_{1}+1$.

Proof. The sufficiency of the conditions, due to Weyl, is usually proved by the minimax principle. It can also be proved using Theorem 5. We have already seen the necessity of $i_{1} \leqq k_{1}$ and $j_{1} \leqq i_{1}$ in the proof of Theorem 4. Now suppose $i_{1}+j_{1} \geqq k_{1}+2$. Let $A=\operatorname{diag}(1$, $\cdots, 1,0, \cdots, 0)$ with $i_{1}-1$ ones, and $B=\operatorname{diag}(0, \cdots, 0,1, \cdots, 1)$ with $j_{1}-1$ ones, where the orders of $A$ and $B$ are $k_{1}$. Since $k_{1}-j_{1}+1 \leqq$ $i_{1}-1$, all the eigenvalues of $A+B$ are $\geqq 1$. Therefore $\gamma_{k_{1}} \geqq 1$, while $\alpha_{i_{1}}=\beta_{j_{1}}=0$, contradicting $\left(i_{1} ; j_{1} ; k_{1}\right) \in S_{1}^{k}$.

THEOREM 8. If $i, j$ and $k$ are ordered pairs of integers satisfying

$$
\begin{aligned}
& 1 \leqq i_{1}<i_{2} \leqq n, \quad 1 \leqq j_{1}<j_{2} \leqq n, \quad 1<k_{1} \leqq k_{2} \leqq n \\
& i_{1}+j_{1} \leqq k_{1}+1 \\
& \left.\begin{array}{l}
i_{1}+j_{2} \\
i_{2}+j_{1}
\end{array}\right\} \leqq k_{2}+1 \\
& i_{1}+i_{2}+j_{1}+j_{2}=k_{1}+k_{2}+3 \text {, }
\end{aligned}
$$

then $(i ; j ; k) \in S_{2}^{n}$.

Proof. By Theorem 4 we may assume $n=k_{2}$. We proceed by 
induction on $n$. If $n=2$ the theorem follows from (1). Suppose the theorem holds for all $n<N$, where $N>2$. By (22), (23) and (24), $i_{p} \leqq k_{p}$ and $j_{p} \leqq k_{p}, p=1,2$. Suppose $i_{1}>1$. Then the pairs $\left(i_{1}-\right.$ $\left.1, i_{2}-1\right),\left(j_{1}, j_{2}\right)$ and $\left(k_{1}-1, k_{2}-1\right)$ satisfy $(22)-(25)$. Therefore by the induction hypothesis $\left(i_{1}-1, i_{2}-1 ; j_{1}, j_{2} ; k_{1}-1, k_{2}-1\right) \in S_{2}^{N-1}$. If we apply Theorem 5 with $u=w=1, v=3$, we find $(i ; j ; k) \in S_{2}^{N}$. A similar method takes care of the case $j_{1}>1$. Therefore we may assume

$$
i_{1}=j_{1}=1 \text {. }
$$

If

$$
\left(i_{1}, i_{2}-1 ; j_{1}, j_{2}-1 ; k_{1}-1, k_{2}-1\right) \in S_{2}^{N-1}
$$

and if

$$
i_{2}+j_{2} \geqq 3+k_{2},
$$

then Theorem 5 with $u=v=2, w=1$ allows us to conclude $(i ; j ; k) \in S_{2}^{N}$. But the Condition (28) which is needed for the application of Theorem 5 will also guarantee (27). To see this, first note that (27) can fail only when

$$
i_{2}=i_{1}+1=2
$$

or

$$
j_{2}=j_{1}+1=2
$$

or

$$
k_{1}=1
$$

or

$$
i_{1}+j_{1}=k_{1}+1 \text {. }
$$

If (i) holds then $i_{2}+j_{2}=2+j_{2} \leqq 2+k_{2}$, contradicting (28). Similarly (ii) cannot hold. If (iii) holds, then by (26), $i_{1}+i_{2}+j_{1}+j_{2}=2+i_{2}+j_{2}=$ $k_{1}+k_{2}+3=k_{2}+4$, or $i_{2}+j_{2}=k_{2}+2$, contradicting (28). Condition (iv) implies (iii) by (26). Therefore we may assume

$$
i_{2}+j_{2} \leqq 2+k_{2} \text {. }
$$

If $i_{2} \geqq k_{1}+2$, it is easy to show by the induction hypothesis that $\left(i_{1}, i_{2}-1 ; j_{1}, j_{2} ; k_{1}, k_{2}-1\right) \in S_{2}^{N-1}$ and Theorem 5 with $u=w=2, v=3$ implies $(i ; j ; k) \in S_{2}^{N}$. Hence we assume

$$
i_{2} \leqq k_{1}+1 \text { and } j_{2} \leqq k_{1}+1 \text {. }
$$

Now (25) and (26) imply $i_{2}+j_{2}=k_{1}+k_{2}+1$, which with (29) implies 
$k_{1}=1$. Therefore by (30) and (22), $i_{2}=j_{2}=2$ and hence $i_{1}=j_{1}=1$. Using (25) we find $k_{2}=2$, contradicting $N>2$. The proof is complete.

If in (25) we replace the equality sign by $\leqq$, Theorem 8 remains true. For if $i, j$ and $k$ satisfy (22)-(24) and the modified (25), there exists a pair $k^{\prime}=\left(k_{1}^{\prime}, k_{2}^{\prime}\right)$ such that $k_{1}^{\prime} \leqq k_{1}, k_{2}^{\prime} \leqq k_{2}$ and $i, j, k^{\prime}$ satisfy (22)-(25). However Theorem 2 suggests that we consider only cases where (19) holds. Conditions (23) and (24) combined may be expressed as follows:

$i_{u}+j_{v} \leqq k_{w}+1$ whenever $1 \leqq u \leqq 2,1 \leqq v \leqq 2,1 \leqq w \leqq 2$, and $u+v=w+1$. This suggests the following conjecture. Let us define inductively the following sequence of sets of triples of sequences of integers: Let $\left(i_{1} ; j_{1} ; k_{1}\right) \in T_{1}^{n}$ if $1 \leqq i_{1} \leqq n, 1 \leqq j_{1} \leqq n, 1 \leqq k_{1} \leqq n$, and $i_{1}+j_{1}=k_{1}+1$, and let $\left(i_{1}, \cdots, i_{r} ; j_{1}, \cdots, j_{r} ; k_{1}, \cdots, k_{r}\right) \in T_{r}^{n}$ if $1 \leqq i_{1}<$ $\cdots<i_{r} \leqq n, 1 \leqq j_{1}<\cdots<j_{r} \leqq n, 1 \leqq k_{1}<\cdots<k_{r} \leqq n$, and

$$
i_{1}+\cdots+i_{r}+j_{1}+\cdots+j_{r}=k_{1}+\cdots+k_{r}+r(r+1) 2,
$$

and

$$
i_{u_{1}}+\cdots+i_{u_{s}}+j_{v_{1}}+\cdots+j_{v_{s}} \leqq k_{w_{1}}+\cdots+k_{w_{s}}+s(s+1) / 2
$$

whenever

$$
(u ; v ; w) \in T_{s}^{r}, 1 \leqq s \leqq r-1 .
$$

Theorem 7 and 8 show that $T_{r}^{n} \subset S_{r}^{n}$ for $r=1,2$. It seems reasonable to conjecture $T_{r}^{n} \subset S_{r}^{n}$ for all $r$. I cannot prove this in general and I know no counterexamples. The case $r=3$ is the following.

THEOREM 9. If $i, j$ and $k$ are ordered triples of integers such that

$$
\begin{aligned}
& 1 \leqq i_{1}<i_{2}<i_{3} \leqq n, 1 \leqq j_{1}<j_{2}<j_{3} \leqq n, 1 \leqq k_{1}<k_{2}<k_{3} \leqq n \\
& i_{1}+j_{1} \leqq k_{1}+1 \\
& \left.\begin{array}{l}
i_{1}+j_{2} \\
i_{2}+j_{1}
\end{array}\right\} \leqq k_{2}+1 \\
& \left.\begin{array}{l}
i_{1}+i_{3} \\
i_{2}+j_{2} \\
i_{3}+j_{1}
\end{array}\right\} \leqq k_{3}+1 \\
& i_{1}+i_{2}+j_{1}+j_{2} \leqq k_{1}+k_{2}+3 \\
& \left.\begin{array}{l}
i_{1}+i_{2}+j_{1}+j_{3} \\
i_{1}+i_{3}+j_{1}+j_{2}
\end{array}\right\} \leqq k_{1}+k_{3}+3 \\
& i_{1}+i_{2}+j_{2}+j_{3} \\
& \left.\begin{array}{l}
i_{2}+i_{3}+j_{1}+j_{2} \\
i_{1}+i_{3}+j_{1}+j_{3}
\end{array}\right\} \leqq k_{2}+k_{3}+3
\end{aligned}
$$




$$
i_{1}+i_{2}+i_{3}+j_{1}+j_{2}+j_{3}=k_{1}+k_{2}+k_{3}+6,
$$

then $(i ; j ; k) \in S_{3}^{n}$.

Proof. The proof begins along the same lines as the proof of Theorem 8 and will only be sketched. We may assume $n=k_{3}$, and proceed by induction on $n$. When $n=3, i_{1}=j_{1}=k_{1}=1, i_{2}=j_{2}=k_{2}=$ $2, i_{3}=j_{3}=k_{3}=3$, and the result follows from (1). Assume the theorem for all $n<N$, where $N>3$. As in Theorem 8, we may assume

$$
i_{1}=j_{1}=1 \text {. }
$$

If

$$
\left(i_{1}, i_{2}-1, i_{3}-1\right),\left(j_{1} j_{2}, j_{3}-1\right),\left(k_{1}-1, k_{2}-1, k_{3}-1\right)
$$

satisfies (33)-(40) and if

$$
i_{2}+j_{3} \geqq k_{3}+3
$$

then the induction hypothesis and Theorem 5 with $u=2, v=3, w=1$ yield the theorem. Again the condition (43) which is needed for the application of Theorem 5 will guarantee (42). For example $k_{1}-1 \geqq 1$, because if $k_{1}=1$, then by (38) and (41), $i_{2}+j_{3} \leqq k_{3}+2$, contradicting (43). The second inequality of (36) together with (43) and $j_{3} \leqq k_{3}$ (which follows from (36)) ensure $j_{3}-1>j_{2}$. We may therefore assume

$$
\left.\begin{array}{l}
i_{2}+j_{3} \\
i_{3}+j_{2}
\end{array}\right\} \leqq k_{3}+2 \text {. }
$$

Next we show that we may assume

$$
i_{2} \leqq k_{1}+1 \text { and } j_{2} \leqq k_{1}+1
$$

by showing that if $i_{2} \geqq k_{1}+2$, then $\left(i_{1}, i_{2}-1, i_{3}-1 ; j_{1}, j_{2}, j_{3} ; k_{1}, k_{2}-1\right.$, $\left.k_{3}-1\right) \in S_{3}^{N-1}$ and Theorem 5 with $u=2, v=3, w=2$ gives $(i ; j ; k) \in S_{3}^{N}$. In a similar manner we may assume

$$
\begin{gathered}
i_{3}+j_{3} \leqq k_{1}+k_{3}+2 \\
i_{3} \leqq k_{2}+1, j_{3} \leqq k_{2}+1 .
\end{gathered}
$$

Now (33)-(41) together with (44)-(47) are easily seen to imply $k_{1}+$ $k_{2}=k_{3}, i_{2}=j_{2}=k_{3}+1, i_{3}=j_{3}=k_{2}+1$ and $k_{1}+1 \leqq k_{2} \leqq 2 k_{1}$. Therefore the theorem will be proved if we can show that

$$
(1, p+1, p+q+1 ; 1, p+1, p+q+1 ; p, p+q, 2 p+q) \in S_{3}^{n}
$$

whenever $1 \leqq q \leqq p$ and $2 p+q=n$.

Let $A, B$ and $A+B$ be of order $n$ with eigenvalues $\left(\alpha_{p}\right),\left(\beta_{p}\right)$ and 
$\left(\gamma_{p}\right)$. We have $q \gamma_{p} \leqq \gamma_{p}+\gamma_{p-1}+\cdots+\gamma_{p-r+1}, q \gamma_{p+q} \leqq \gamma_{p+q}+\cdots+\gamma_{p+1}$, and $q \gamma_{2 p+q} \leqq \gamma_{2 p+q}+\cdots+\gamma_{2 p+1}$. Hence

$$
\begin{aligned}
q\left(\gamma_{p}+\gamma_{p+q}+\gamma_{2 p+q}\right) & \leqq \operatorname{trace}(A+B) \\
& -\left(\gamma_{1}+\cdots+\gamma_{p-q}+\gamma_{p+q+1}+\cdots+\gamma_{2 p}\right) .
\end{aligned}
$$

Similarly

$$
\begin{aligned}
q\left(\alpha_{1}+\alpha_{p+1}\right. & \left.+\alpha_{p+q+1}\right) \\
& \geqq \operatorname{trace} A-\left(\alpha_{q+1}+\cdots+\alpha_{p}+\alpha_{p+2 q+1}+\cdots+\alpha_{2 p+q}\right)
\end{aligned}
$$

and we have a similar statement for the $\beta$ 's. Therefore we need only prove

$$
\begin{aligned}
& (q+1, \cdots, p, p+2 p+1, \cdots, 2 p+q ; q+1, \cdots, p, p+2 q \\
& \quad+1, \cdots, 2 p+q ; 1, \cdots, p-q, p+q+1, \cdots, 2 p) \in \widetilde{S}_{2 p-2 q}^{n} .
\end{aligned}
$$

This will follows from Theorem 3 (ii) if we can show

$$
\begin{aligned}
& (1, \cdots, p-q, p+q+1, \cdots, 2 p ; 1, \cdots, p-q, p+q \\
& \quad+1, \cdots, 2 p ; q+1, \cdots, p, p+2 q+1, \cdots, 2 p+q) \in S_{2 p-2 q}^{n} .
\end{aligned}
$$

By Theorem 6 we have

$$
\begin{array}{r}
(1, \cdots, 2 p-2 q ; 1, \cdots, p-q, p+1, \cdots, 2 p-q ; \\
1, \cdots, p-q, p+1, \cdots, 2 p-q) \in S_{2 p-2 q}^{2 p-q} .
\end{array}
$$

We may apply Theorem $5 q$ times with $u=w=p-q+1, v=2 p-2 q+$ 1 to obtain

$$
\begin{aligned}
& (1, \cdots, p-q, p+1, \cdots, 2 p-q ; 1, \cdots, p-q \\
& \quad p+1, \cdots, 2 p-q ; 1, \cdots, p-q, p+q+1, \cdots, 2 p) \in S_{2 p-2 q}^{2 p} .
\end{aligned}
$$

Theorem 5 applied $q$ times with $u=v=p-q+1, w=1$ yields (48). The proof is now complete.

A proof of $T_{4}^{n} \subset S_{4}^{n}$ along the same lines runs into the following difficulty. The first half of the proof, that is, the application of Theorem 5 in all possible ways, carries through. However the cases left untouched turn out to be too numerous to handle by the methods of the second half of the proof of Theorem 9. I have verified $T_{4}^{n} \subset S_{4}^{n}$ for $n \leqq 8$.

As for the statement $S_{r}^{n} \subset T_{r}^{n}$, it is possible to show by a consideration of diagonal matrices that if $(i ; j ; k) \in S_{r}^{n}$ then (32) holds for $s=1,2$. This together with the remark following Theorem 8 determines $S_{2}^{n}$. But the general statement $S_{r}^{n} \subset T_{r}^{n}$ is false even if we weaken the definition of $T_{r}^{n}$ by replacing the equality sign in (31) by $\leqq$. For example a consideration of the trace condition shows that $(1,5,9,12 ; 1,5,9,12 ; 4,8,12$, 16) $\in S_{4}^{16}$.

Guided by Theorem 3 (ii), the dual set $\widetilde{T}_{r}^{n}$ may be defined inductively 
as follows: $\left(i_{1}, j_{1}, k_{1}\right) \in \widetilde{T}_{1}^{n}$ if $i_{1}+j_{1}=k_{1}+n$, and $(i ; j ; k) \in \widetilde{T}_{r}^{n}$ if $i_{1}+\cdots+$ $i_{r}+j_{1}+\cdots+j_{r}=k_{1}+\cdots+k_{r}+n r-r(r-1) / 2$ and

$$
i_{u_{1}}+\cdots+i_{u_{s}}+j_{v_{1}}+\cdots+j_{v_{s}} \geqq k_{w_{1}}+\cdots+k_{w_{s}}+n s-s(s-1) / 2
$$

whenever $(u ; v ; w) \in \widetilde{T}_{s}^{r}$. It is easily seen that $(i ; j ; k) \in T_{r}^{n}$ if and only if $\left(n-i_{r}+1, \cdots, n-i_{1}+1 ; n-j_{r}+1, \cdots, n-j_{1}+1 ; n-k_{r}+1, \cdots\right.$, $\left.n-k_{1}+1\right) \in \widetilde{T}_{r}^{n}$. Hence by Theorem $3, T_{r}^{n} \subset S_{r}^{n}$ is equivalent to $\widetilde{T}_{r}^{n} \subset$ $\widetilde{S}_{r}^{n}$. I have been unable to prove the analogue of the last transformation rule of Theorem 3. However I can prove that if $(i ; j ; k) \in \widetilde{T}_{1}^{n}$, then $\left(i^{\prime} ; j^{\prime} ; k^{\prime}\right) \in T_{n-1}^{n}$, where $i^{\prime}, j^{\prime}$, and $k^{\prime}$ are the complements with respect to $n$.

3. The set $E$. We return to the problem of determining the set $E$ defined in the introduction. Let $F$ be the set of points $\gamma$ defined by $\gamma_{1} \geqq \cdots \geqq \gamma_{n}$,

$$
\gamma_{1}+\cdots+\gamma_{n}=\alpha_{1}+\cdots+\alpha_{n}+\beta_{1}+\cdots \beta_{n},
$$

and

$$
\gamma_{k_{1}}+\cdots+\gamma_{k_{r}} \leqq \alpha_{i_{1}}+\cdots+\alpha_{i_{r}}+\beta_{j}+\cdots+\beta_{j_{r}}
$$

whenever

$$
(i ; j ; k) \in T_{r}^{n}, 1 \leqq r \leqq n-1 .
$$

In $\S 2$ we have shown that $E \subset F$ for $n \leqq 4$. In this section we will prove that $E=F$ for $n \leqq 4$.

There is no loss of generality in assuming $\alpha_{1}>\cdots>\alpha_{n}$ and $\beta_{1}>$ $\cdots>\beta_{n}$. The set $E^{\prime}$ defined in $\S 1$ is a closed subset of $E$. Since $F$ is closed and convex, it will follow that $E^{\prime}=F$, and therefore $E=F$, if the boundary of $E^{\prime}$ is contained in the boundary of $F$. To see this, let $\gamma$ be an interior point of $E^{\prime}$ and suppose $\gamma^{\prime}$ is any point of $F$. If $\gamma^{\prime}$ is not in $E^{\prime}$ there must be a boundary point of $E^{\prime}$ in the open segment joining $\gamma$ and $\gamma^{\prime}$. But all points of this open segment are interior points of $F$.

A boundary points of $E^{\prime}$ with at least two equal coordinates is obviously a boundary point of $F$. If $\gamma$ is a boundary point of $E^{\prime}$ with distinct coordinates, there is associated with $\gamma$ a triple $(i ; j ; k)$ satisfying the conditions of Theorem 2. All that remains to prove is that $(i ; j ; k)$ $\in T_{r}^{n}$. To this end we first prove the following theorem.

THEOREM 10. If $\gamma$ is a boundary point of $E^{\prime}$ with associated sequences $(i ; j ; k)$ of order $r$, then for any $(x ; y ; z) \in \widetilde{S}_{m}^{r}$, there cannot exist a triple $(u ; v ; w) \in S_{m}^{n-r}$ such that $i_{x_{p}} \leqq x_{p}+u_{p}-1, j_{y_{p}} \leqq y_{p}+v_{p}-$ 1 , and $k_{z_{p}} \geqq z_{p}+w_{p}$, for $1 \leqq p \leqq m$. 
Proof. For convenience, we write $\alpha(p)$ instead of $\alpha_{p}$. By hypothesis there exist Hermitian matrices $A_{1}, B_{1}$, and $A_{1}+B_{1}$ with eigenvalues $\left(\alpha\left(i_{p}\right)\right),\left(\beta\left(j_{p}\right)\right)$, and $\left(\gamma\left(k_{p}\right)\right), p=1, \cdots, r$, and Hermitian matrices $A_{2}, B_{2}$, and $A_{2}+B_{2}$ with eigenvalues $\left(\alpha\left(i_{p}^{\prime}\right)\right),\left(\beta\left(j_{p}^{\prime}\right)\right)$, and $\left(\gamma\left(k_{p}^{\prime}\right)\right), p=1, \cdots, n-r$, where $i^{\prime}$ is complement of $i$ with respect to $n$. If there exists a triple $(u ; v, w) \in S_{m}^{n-r}$ such that $i_{x_{p}}<i_{u_{p}}^{\prime}, j_{y_{p}}<j_{v_{p}}^{\prime}$, and $k_{z_{p}}^{\prime}>k_{w_{p}}^{\prime}, 1 \leqq p \leqq m$, then we have

$$
\sum_{p=1}^{m} \alpha\left(i_{x_{p}}\right)+\sum_{p=1}^{m} \beta\left(j_{y_{p}}\right) \leqq \sum_{p=1}^{m} \gamma\left(k_{z_{p}}\right)<\sum_{p=1}^{m} \gamma\left(k_{w_{p}}^{\prime}\right) \leqq \sum_{p=1}^{m} \alpha\left(i_{u}^{\prime}\right)+\sum_{p=1}^{m} \beta\left(j_{v}^{\prime}\right) .
$$

This is impossible since $\alpha\left(i_{x_{p}}\right)>\alpha\left(i_{u_{p}}^{\prime}\right)$ and $\beta\left(j_{y_{p}}\right)<\beta\left(j_{v_{p}}^{\prime}\right)$. Therefore it remains only to show that $i_{p}<i_{q}^{\prime}$ is implied by $i_{p} \leqq p+q-1$. If $i_{p} \leqq$ $p+q-1$, then at least $p$ terms of the sequence $i$ are $\leqq p+q-1$. Therefore at most $q-1$ positive integers $\leqq p+q-1$ are not in $i$. Hence $i_{q}^{\prime}>p+q-1 \geqq i_{p}$.

THEOREM 11. If $\gamma$ is a boundary point of $E^{\prime}$ with associated sequences $i, j, k$ of order $r$, then $i_{x}+j_{y} \geqq k_{z}+r$ whenever $(x, y, z) \in \widetilde{T}_{1}^{r}$. More generally, if $x+y \geqq z+r$, the $i_{x}-x+j_{y}-y \geqq k_{z}-z$.

Proof. We have $n \geqq r+1 \geqq 2$. Since $(x ; y ; x+y-r) \in \widetilde{T}_{1}^{r} \subset \widetilde{S}_{1}^{r}$, it follows that $(x ; y ; z) \in \widetilde{S}_{1}^{r}$. Let $u=i_{x}-x+1, v=j_{y}-y+1$, and $w=k_{z}-z$. Clearly, $u \geqq 1, v \geqq 1$, and $w \leqq n-r$ since $k_{1}-1 \leqq k_{2}-$ $2 \leqq \cdots \leqq k_{r}-r \leqq n-r$. We must prove $u+v \geqq w+2$. If $u+v \leqq$ $w+1$, then $w \geqq 1, u \leqq w$, and $v \leqq w$. Therefore $(u ; v ; w) \in T_{1}^{n-r}$. This contradicts Theorem 10 .

THeOREM 12. Under the same hypothesis as Theorem 11, if $n \geqq r+$ 2 , then $i_{x_{1}}+i_{x_{2}}+j_{y_{1}}+j_{y_{2}} \geqq k_{z_{1}}+k_{z_{2}}+2 r-1$ whenever $(x, y, z) \in \widetilde{T}_{2}^{r}$.

Proof. We are given $x_{1}+y_{2} \geqq z_{1}+r, x_{2}+y_{1} \geqq z_{1}+r, x_{2}+y_{2} \geqq$ $z_{2}+r$, and $x_{1}+x_{2}+y_{1}+y_{2}=z_{1}+z_{2}+2 r-1$. Let $a_{p}=i_{x_{p}}-x_{p}+1$, $b_{p}=j_{y_{p}}-y_{p}+1$, and $c_{p}=w_{z_{p}}-z_{p}, p=1,2$. By Theorem 11, $a_{1}+b_{2} \geqq$ $c_{1}+2, a_{2}+b_{1} \geqq c_{1}+2$, and $a_{2}+b_{2} \geqq c_{2}+2$. Suppose the theorem fails. Then $a_{1}+a_{2}+b_{1}+b_{2} \leqq c_{1}+c_{2}+3$. Therefore

$$
\begin{aligned}
& a_{1}+b_{1} \leqq c_{1}+1 \\
& a_{1}+b_{2} \leqq c_{2}+1 \\
& a_{2}+b_{1} \leqq c_{2}+1 .
\end{aligned}
$$

Also $1 \leqq a_{1} \leqq a_{2}, 1 \leqq b_{1} \leqq b_{2}$, and $c_{2} \leqq n-r$. By (49), $c_{1} \geqq 1$. Moreover $c_{1}+2 \leqq a_{1}+b_{2} \leqq c_{2}+1$, so that $c_{1}+1 \leqq c_{2}$. Now let $u_{1}=a_{1}, u_{2}=$ $\max \left(a_{2}, a_{1}+1\right), v_{1}=b_{1}, v_{2}=\max \left(b_{2}, b_{1}+1\right), w_{1}=c_{1}$, and $w_{2}=c_{2}$. It is easy to see that $u_{1}+v_{1} \leqq w_{1}+1, u_{1}+v_{2} \leqq w_{2}+1, u_{2}+v_{1} \leqq w_{2}+1$, and 
$u_{1}+u_{2}+v_{1}+v_{2} \leqq w_{1}+w_{2}+3$. As previously remarked there exists a pair $\left(w_{1}^{\prime}, w_{2}^{\prime}\right)$ such that $w_{1}^{\prime} \leqq w_{1}, w_{2}^{\prime} \leqq w_{2}$, and $(u ; v ; w) \in T_{2}^{n-r}$. This contradicts Theorem 10.

Using a generalized version of Theorem 12, it is possible to show that

$$
i_{x_{1}}+i_{x_{2}}+i_{x_{3}}+j_{y_{1}}+j_{y_{2}}+j_{y_{3}} \geqq k_{z_{1}}+k_{z_{2}}+k_{z_{3}}+r+r-1+r-2
$$

whenever $(x ; y ; z) \in \widetilde{T}_{3}^{r}, n \geqq r+2$.

THEOREM 13. If $\gamma$ is a boundary point of $E^{\prime}$ with associated sequences $i, j, k$ of order $r=1,2,3$ or $n-1$, then $(i ; j ; k) \in T_{r}^{n}$.

Proof. For $r=1$ this is obvious. For $r=n-1$, the complementary sequences with respect to $n$ are of order 1 and satisfy $i_{1}^{\prime}+j_{1}^{\prime}=k_{1}^{\prime}+n$. Therefore $\left(i^{\prime} ; j^{\prime} ; k^{\prime}\right) \in \widetilde{T}_{1}^{n}$. By the last sentence of $\S 2$, it follows that $(i ; j ; k) \in T_{n-1}^{n}$. For the cases $n=3,4$ this can be easily verified by listing cases. Now suppose $r=2$. We must prove that (23) and (24) hold. In view of (25), this means we must show that $i_{x}+j_{y} \geqq k_{z}+2$ whenever $(x ; y ; z) \in \widetilde{T}_{1}^{2}$. But this follows from Theorem 11 . Suppose $r=3$. We may assume $n \geqq 5$. By (40) and Theorems 11 and 12 we have (34)-(39), since if $(x ; y ; z) \in \widetilde{T}_{p}^{3}$ then $\left(x^{\prime} ; y^{\prime} ; z^{\prime}\right) \in T_{3-q}^{3}, p=1,2$.

Theorem 13 completes the proof that $E=F$ for $n \leqq 4$. It is possible to extend the proof to $n \leqq 8$. But the general case remains open.

\section{REFERENCES}

1. A. R. Amir-Moez, Extreme properties of eigenvalues of a Hermitian transformation and singular values of the sum and product of linear transformations, 23 (1955), 463-476. 2. G. R. Gantmacher, Matrizenrechnung II, VEB Deutcher Verlag der Wissenschaften, Berlin 1959.

3. V. B. Lidskii, On the characteristic numbers of the sum and product of symmetric matrices (in Russian), Doklady Akad. Nauk SSSR, 72 (1950), 769-772.

4. H. Weyl, Das asymptotische Verteilungsgesetz der Eigenwerte linearer partieller Differentialgleichungen, Math. Annalen, 71 (1912), 441-479.

5. W. Wielandt, An extremum property of sums of eigenvalues, Proc. Amer. Math., Soc., 6 (1955), 106-110.

UNIVERSITY OF CALIFORNIA

Los Angeles, California 



\section{PACIFIC JOURNAL OF MATHEMATICS}

\section{EDITORS}

Ralph S. Phillips

Stanford University

Stanford, California

M. G. Arsove

University of Washington

Seattle 5, Washington
A. L. Whiteman

University of Southern Californla

Los Angeles 7, California

Lowell J. Paige

University of California

Los Angeles 24, California

\section{ASSOCIATE EDITORS}

E. F. BECKENBACH

D. DERRY

H. L. ROYDEN

E. G. STRAUS

T. M. CHERRY

M. OHTSUKA

E. SPANIER

F. WOLF

\section{SUPPORTING INSTITUTIONS}

UNIVERSITY OF BRITISH COLUMBIA

STANFORD UNIVERSITY

CALIFORNIA INSTITUTE OF TECHNOLOGY

UNIVERSITY OF CALIFORNIA

MONTANA STATE UNIVERSITY

UNIVERSITY OF TOKYO

UNIVERSITY OF UTAH

UNIVERSITY OF NEVADA

NEW MEXICO STATE UNIVERSITY

OREGON STATE UNIVERSITY

UNIVERSITY OF OREGON

OSAKA UNIVERSITY

WASHINGTON STATE UNIVERSITY

UNIVERSITY OF WASHINGTON

UNIVERSITY OF SOUTHERN CALIFORNIA

AMERICAN MATHEMATICAL SOCIETY CALIFORNIA RESEARCH CORPORATION SPACE TECHNOLOGY LABORATORIES NAVAL ORDNANCE TEST STATION 


\section{Pacific Journal of Mathematics}

\section{Vol. 12, No. $1 \quad$ January, 1962}

Jonathan L. Alperin, Groups with finitely many automorphisms $\ldots \ldots \ldots \ldots \ldots \ldots \ldots \ldots$

Martin Arthur Arkowitz, The generalized Whitehead product ................ 7

John D. Baum, Instability and asymptoticity in toplogical dynamics . . . . . . . . . . 25

William Aaron Beyer, Hausdorff dimension of level sets of some Rademacher series .... $\quad 35$

Frank Herbert Brownell, III, A note on Cook's wave-matrix theorem . . . . . . . . . . . . . 47

Gulbank D. Chakerian, An inequality for closed space curves ................. 53

Inge Futtrup Christensen, Some further extensions of a theorem of Marcinkiewicz ....... 59

Charles Vernon Coffman, Linear differential equations on cones in Banach spaces . . . . . 69

Eckford Cohen, Arithmetical notes. III. Certain equally distributed sets of integers . . . . . 77

John Irving Derr and Angus E. Taylor, Operators of meromorphic type with multiple poles

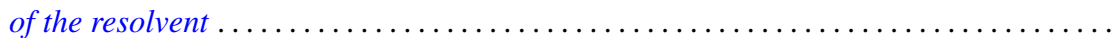

Jacob Feldman, On measurability of stochastic processes in products space .............

Robert S. Freeman, Closed extensions of the Laplace operator determined by a general class of boundary conditions, for unbounded regions ......................

Robert E. Fullerton, Geometric structure of absolute basis systems in a linear topological

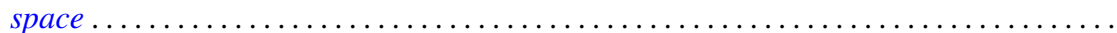

Dieter Gaier, On conformal mapping of nearly circular regions

Andrew Mattei Gleason and Hassler Whitney, The extension of linear functionals defined

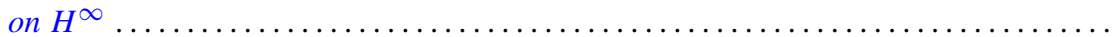

Seymour Goldberg, Closed linear operators and associated continuous linear

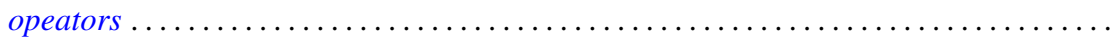

Basil Gordon, Aviezri Siegmund Fraenkel and Ernst Gabor Straus, On the determination of sets by the sets of sums of a certain order

Branko Grünbaum, The dimension of intersections of convex sets. .

Paul Daniel Hill, On the number of pure subgroups

Robert Peter Holten, Generalized Goursat problem . .

Alfred Horn, Eigenvalues of sums of Hermitian matrices ...........

Henry C. Howard, Oscillation and nonoscillation criteria for

$$
y^{\prime \prime}(x)+f(y(x)) p(x)=0
$$

Taqdir Husain, $S$-spaces and the open mapping theorem ...

Richard Eugene Isaac, Markov processes and unique stationary probability measures ...

John Rolfe Isbell, Supercomplete spaces ....................

John Rolfe Isbell, On finite-dimensional uniform spaces. II .........

N. Jacobson, A note on automorphisms of Lie algebras ..............

Antoni A. Kosinski, A theorem on families of acyclic sets and its applications

Marvin David Marcus and H. Minc, The invariance of symmetric functions of singular values...

Ralph David McWilliams, A note on weak sequential convergence.

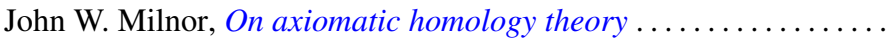

Victor Julius Mizel and Malempati Madhusudana Rao, Nonsymmetric projections in

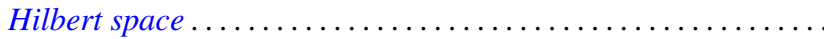

Calvin Cooper Moore, On the Frobenius reciprocity theorem for locally compact

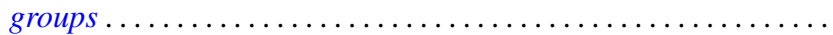

Donald J. Newman, The Gibbs phenomenon for Hausdorff means . 